\title{
COMUNIDADES DE TRICÓPTEROS (TRICHOPTERA) DE AGUA CORRIENTE EN LA PROVINCIA DE CÁDIZ
}

\author{
Antonio Ruiz Garcia \\ IES Álvar Núñez. Poligono San Benito s/n. 11407 Jerez de la Frontera. Cádiz
}

\begin{abstract}
RESUMEN
En los ríos del SO de la peninsula Ibérica se han han encontrado seis asociaciones de especies deTricópteros. Asociacion I: Rh. fonticola, Sc. festiva y T. waeneri; Asociacion II: S. aurata, C. marsupus, H. infernalis, St. crossotus y S. vittatum; Asociacion III: P. kingi, Rh. munda, $H$. instabilis y A. reducta; Asociacion IV: Ch. marginata, $H$. cf punica y $S$. argentipunctellus; Asociacion V: H. vectis y H. lobata y Asociación VI: Ch. lepida, H. exocellata, P. vandeli, E. deceptor, C. dissimilis y $\mathrm{C}$. monserrati. A continuación se clasifican los cursos de agua estudiados y se discuten los requerimientos ecológicos de cada grupo de especies.
\end{abstract}

Palabras clave: Comunidad, Tricópteros, SO Peninsula Ibérica.

\begin{abstract}
Six trichoptera species associations were found in the running waters of the $S W$ of the Iberian Peninsula. Association I: Rh. fonticola, Sc. festiva and T. waeneri; Association II: S. aurata, C. marsupus, St. crossotus and S. vittatum; Association 111: P. kingi, Rh. munda, H. instabilis and A. reducta; Association IV: Ch. marginata, H. ef punica and $\mathrm{S}$. argentipunctellus; Association V. H. vectis and $\mathrm{H}$. lobata and Association VI: Ch. lepida, H. exocellata, P. vandeli, E. deceptor, C. dissimilis and C. monserrati. Next, the studied watercourses are classified and the ecological demands of each species group are analysed.
\end{abstract}

Keywords:Community, Trichoptera, SW Iberian Peninsula

\section{INTRODUCCIÓN}

Los tricópteros constituyen una fracción importante del bentos fluvial. Por este motivo, el conocimiento de este grupo es fundamental a la hora de interpretar el funcionamiento de los cursos de agua. Actuan como integradores de condiciones ambientales y desempeñan un importante papel en la transformacion y transferencia de materia y energia dentro del ecosistema acuático (Basaguren \& Orive, 1991). Proporcionan por si solos la imagen de la estructura ecológica de un sistema acuático (Bournaud et al., 1980).

El estudio de los macroinvertebrados acuaticos ha experimentado un gran dessarrollo en las últimas décadas, aunque este dessarrollo no ha sido homogéneo en toda la Peninsula Ibérica (Alba-
Tercedor et al.,1992). Este panorama define la situación actual con respecto al conocimiento de los tricopteros ibéricos, con una mitad norte peninsular bien estudiada y una mitad meridional peor conocida (Gonzalez et al., 1987).

En el ámbito territorial en el que se circunscribe el presente trabajo (provincia de Cádiz) han sido escasos los estudios dedicados a este grupo faunistico, destacando los trabajos faunisticos (Garcia de Jalón, 1982; Gonzalez et al., 1990; Ruiz, 1998) y taxonómicos (Malicky, 1979 y 1982; González \& Malicky, 1988; González \& Iglesias, 1989). Por lo que respecta a trabajos limnológicos sólo ha sido estudiada la cabecera del rio Guadalete (Gallardo-Mayenco, 1991, 1993 y 1994).

En este trabajo se presentan datos acerca de la composición y la tipologia de las comunidades de 


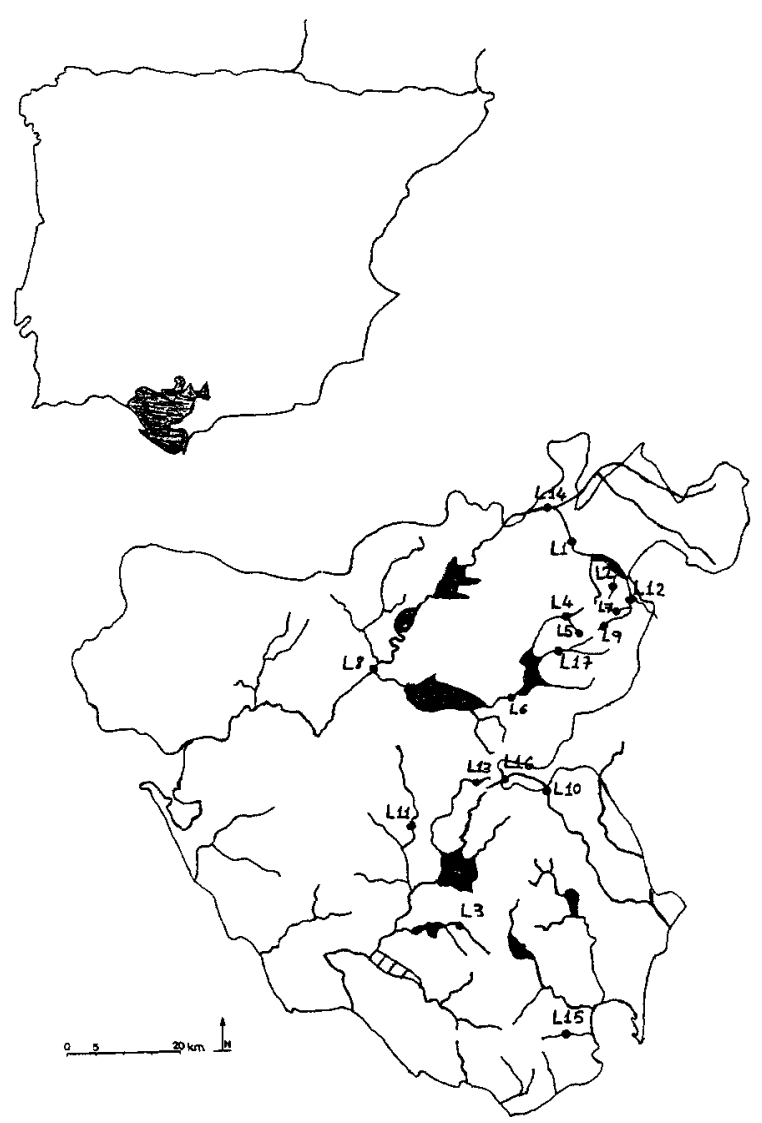

Figura 1. Area de estudio y localización de las unidades de muestreo. Study area and sampling sites location.

tricopteros que pueblan los cursos de agua mas representativos de la provincia de Cadiz.

\section{AREA DE ESTUDIO}

$\mathrm{El}$ area escogida para este estudio ha sido un conjunto de cuencas situadas en el Suroeste de la Peninsula Ibérica, que en su conjunto constituyen la totalidad de la provincia de Cádiz (Fig. 1). Son cursos de agua tipicamente Mediterráneos sujetos a importantes fluctuaciones de caudal a 10 largo del año. Las principales cuencas son la del río Guadalete con $3677 \mathrm{Km}^{2}$ de superficie y Barbate con una extensión de $1300 \mathrm{Km}^{2}$, que representan cl $75 \%$ del territorio estudiado. La superficie restante es drenada por las subcuencas del rio Hozgarganta, Guadarranque, Palmones y rio de la Miel, principalmente. Con respecto al uso del suelo, la cabecera de todos estos cauces esta constituida por terreno forestal en buen estado de conservacibn; el curso medio es terreno de campiña dedicado a la agricultura y ganaderia; por último, en las zonas de desembocadura es donde se concentra la mayoria de la población provocando serios problemas de contaminación.

Desde un punto de vista geológico, la zona está claramente separada en una mitad norte con predominio de materiales calizos, dolomiticos, margo-yesíferos y calcareniticos y una mitad sur donde predominan las areniscas del Aljibe. Esta constitución geologica provoca una marcada diferenciación en la mineralización (valores de conductividad) de las aguas de la cuenca del Guadalete con respecto a las demas. No obstante, algunas formaciones diapiricas son responsables de la existencia de manantiales salinos en la cuenca del rio Barbate, por lo que existen arroyos de carácter salino (cond. superior a 8 $\mathrm{mS} / \mathrm{cm}$ ) (Tabla 1).

\section{MATERIAL Y MÉTODOS}

Dentro del área de estudio se escogieron 17 localidades representativas del total de los cursos de agua. Cada una de ellas fue visitada en dos ocasiones en primavera y verano de 1995. Los muestreos se realizaron con trampas de luz negra, para la captura de adultos, que se colocaban al anochecer durante un periodo de 1.5 horas cerca del agua en los tramos seleccionados. Las hembras del género Hydropsyche se repartieron en las distintas especies segdn la proporción en la que aparecian los machos en cada muestreo. Las variables fisico-quimicas que aperecen en la figura 1 fueron tomadas en Junio del mismo año, excepto la estación L9 analizada en Abril.

En el tratamiento estadistico de los datos sólo se tuvieron en cuenta aquellas especies cuya abundancia por unidad de esfuerzo superaba el $5 \%$ del total para cada localidad, obteniéndose una matriz de 23 especies - 17 localidades. La 
Tabla 1. Valores de los parametros físico-químicos medidos en el área de estudio. C: cantos; Cr: gravas; F: finos. Phpsico-chemical parameter in the study urea. C: stones; Gr: gravels; F: fines.

\begin{tabular}{|c|c|c|c|c|c|c|c|c|c|c|}
\hline $\mathbf{N}^{0}$ & Localidad & $\mathbf{P H}$ & T. ${ }^{0} \mathrm{C}$ & $\begin{array}{l}\text { Cond. } \\
\mathrm{mS} / \mathrm{cm}\end{array}$ & $\begin{array}{l}\text { Veloc. } \\
\text { corr. }\end{array}$ & $\begin{array}{c}\text { Altitud } \\
\text { m }\end{array}$ & Sustrato & $\begin{array}{l}\text { Dist. orig. } \\
\text { Km }\end{array}$ & $\begin{array}{c}\text { Diversidad } \\
\text { H }\end{array}$ & $\begin{array}{c}\text { Riqueza } \\
\text { esp. }\end{array}$ \\
\hline $\mathrm{Ll}$ & Algodonales & 7.7 & 20 & 0.97 & moderada & 320 & $\mathrm{C}, \mathrm{Gr}$ & 28 & 2.50 & 13 \\
\hline L2 & Arroyomolinos & 7 & 17 & 2.6 & moderada & 3.50 & $\mathrm{C}, \mathrm{Gr}, \mathrm{F}$ & 2.5 & 2.90 & 14 \\
\hline L3 & Benalud & 6.6 & 21 & 0.27 & baja & 60 & $\mathrm{C}, \mathrm{Gr}$ & 6 & 2.07 & 6 \\
\hline $\mathrm{L} 4$ & Benamahoma & 7.6 & 16 & 0.52 & alta & 420 & $\mathrm{C}, \mathrm{Gr}$ & 0.3 & 3.82 & 15 \\
\hline L5 & Descansadero & 6.9 & 15 & 0.61 & alta & 600 & $\mathrm{C}, \mathrm{Gr}$ & 0.02 & 2.41 & 12 \\
\hline L6 & Hurones & 7.6 & 22 & 0.75 & moderada & 160 & $\mathrm{C}, \mathrm{Gr}$ & 22 & I.88 & 10 \\
\hline L7 & Gaiddvar & 7.8 & 17 & 0.6 .5 & alta & 660 & $\mathrm{C}, \mathrm{Gr}$ & 0.5 & 3.07 & 12 \\
\hline L8 & J. de los ríos & 7.9 & 26 & 1.37 & baja & 35 & $\mathrm{Gr}, \mathrm{F}$ & 140 & 0.30 & 3 \\
\hline L9 & Nacimiento & 6.9 & 13 & 0.6 .5 & baja & 1100 & $\mathrm{C}, \mathrm{Gr}$ & 0.2 & 1,41 & 4 \\
\hline $\mathrm{L} 10$ & Pte. Cañillas & 7.3 & 22 & 1.08 & moderada & 170 & $\mathrm{C}, \mathrm{Gr}$ & 14 & 3.32 & 18 \\
\hline $\mathrm{L} 11$ & R. Álamo & 7.7 & 24 & 8.7 & baja & 20 & $\mathrm{~F}$ & 9 & 0.48 & 2 \\
\hline L12 & Pte. Terrona & 7.8 & 22 & 1.11 & moderada & 350 & $C, \mathrm{Gr}$ & 8 & 2.79 & 27 \\
\hline L13 & Pto. Oscuro & 6.3 & 18 & 0.20 & alta & 380 & $C, \mathrm{Gr}$ & 3 & 2.33 & 15 \\
\hline L14 & Puerto Serrano & 7.9 & 23 & 0.98 & moderada & 270 & $\mathrm{C}, \mathrm{Gr}$ & 43 & 2.08 & 9 \\
\hline $\mathrm{L} 15$ & R. de la miel & 6.4 & 18 & 0.25 & alta & 200 & $\mathrm{C}, \mathrm{Gr}$ & 4 & 3.18 & 14 \\
\hline L16 & La Sauceda & 6.8 & 19 & 0.30 & alta & 520 & $\vec{C}, \mathrm{Gr}$ & 1.5 & 2.72 & 16 \\
\hline L17 & Tavizna & 7.7 & 20 & 1.30 & moderada & 360 & $\mathrm{C}, \mathrm{Gr}$ & 11 & & \\
\hline
\end{tabular}

ordenación de las localidades en el gradiente ambiental se obtuvo a partir de un Análisis de Componentes Principales (PCA) de la matriz de variables fisico-quimicas y biológicas Para detectar las afinidades entre inventarios se agmparon las estaciones de forma jerárquica por el método de la media a partir de la matriz de correlaciones obtenida de la matriz de datos especies-localidades. Las agrupaciones de especies tambien se establecieron de forma jerárquica por el método de la media. Los grupos se formaron a partir de la matriz de correlaciones. Para analizar los posibles gradientes ambientales que determinan la distribución de los diferentes taxones se realizó un segundo PCA sobre la matriz de datos especies localidades. Todas las variables originales fueron transformadas con la función $\log (x+1)$.

\section{RESULTADOS}

En la figura 2 aparece la relación de las variables fisico-quimicas y biológicas con las estaciones de muestreo obtenida tras la aplicación del PCA

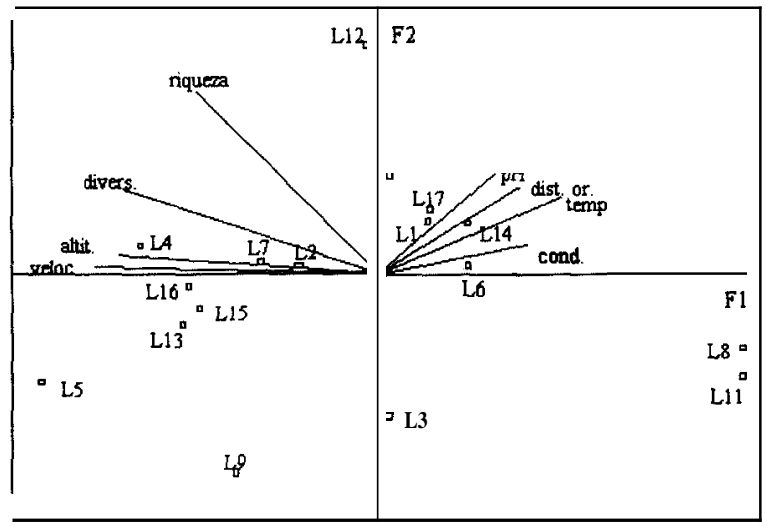

Figura 2. Representación de la situación de los parámetros físicoyuimicos y localidades en el espacio definido por los dos primeros ejes del PCA (biplot). Physico-chemical parameters and sampling site location in the space defined y the first two axis of the Principul Components Analysis (PCA) biplot.

para los dos primeros ejes. Conjuntamente acumulan e $172.2 \%$ de la variación. El primer factor explica el $54.4 \%$ de la varianza, el segundo el $17.8 \%$. 
La distribución de las unidades de muestreo en funcion del primer factor (F1) implica una separación de las muestras de cabecera ligadas a su extremo negativo de las de los tramos más bajos asociadas a su extremo positivo. Podemos apreciar que F1 esta correlacionado positivamente con variables como $\mathrm{PH}$, temperatura, conductividad y distancia al origen, atributos que definen los tramos bajos de los rios; y está negativamente relacionado con la altitud, diversidad, riqueza especifica y velocidad de la corriente, parámetros que van aumentando conforme nos desplazamos aguas arriba. Todo esto sugiere que F1 representa un gradiente altitudinal y de mineralizacion.

El segundo eje (F2) tiene poca significación real ya que sólo tiene variables asociadas en el extremo positivo. Discrimina las estaciones L12 y L9 entre si, dos estaciones del mismo rio.

En concreto, una cabecera ácida con escasa riqueza especifica ( L9) de la estacidn con la maxima riqueza especifica ( L12).

La ordenación de las estaciones de muestreo en funcion de su composicidn faunistica se muestra en la figura 3. A la vista del dendrograma resultante los tramos estudiados pueden agruparse en cuatro categorias: Arroyos de cabecera, tramos altos, curso medio del Guadalete y tramos bajos.

En el primer grupo las mayores correlaciones se dan entre las estaciones L16 y L13 por un lado y L5 y L7 por otro, separando los arroyos que discurren por sustrato siliceo ( Sierra del Aljibe) de

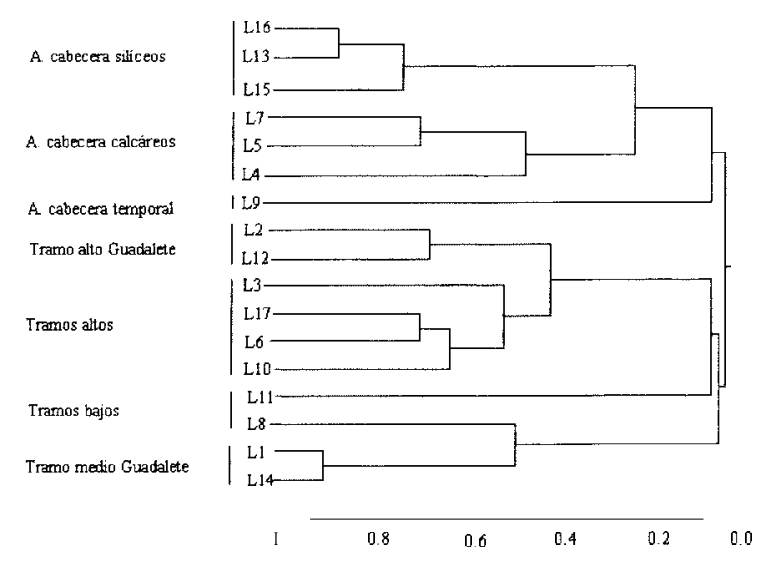

Figura 3. Dendrograma obtenido para la agrupación de las localidades. Clustering diagram of sampling points. los que circulan por calizas ( Sierra de Grazalema). El nacimiento del Guadalete ( L9 ) presenta escasa afinidad con los demas tramos debido, posiblemente, a su carácter temporal.

En las localidades correspondientes a tramos altos las mejores correlaciones se producen entre las estaciones L2 y L12 y L6 y L17. Es de destacar la mayor afinidad de la estacidn L2 (Arroyomolinos) con este grupo de estaciones que con las anteriores. Es posible que la contaminación observada sea la responsable de que este curso de agua se parezca mas al curso alto del Guadalete que a los arroyos de cabecera.

Las estaciones L1 y L14 representan el curso medio del Guadalete. Son localidades muy homogéneas desde un punto de vista físicoquimico ( Fig. 2 ) y biológico que muestran la mayor correlación de todo el conjunto.

Por ultimo , las estaciones L8 y L11 se corresponden con el curso bajo del Guadalete y el rio de los Alamos respectivamente. Estos cursos están caracterizados por la elevada temperatura y conductividad de sus aguas (Fig. 2 ).

Basándonos en el dendrograma de la figura 4, seis son los grupos de especies que quedan más claramente definidos. El patrón de asociación fue el siguiente: Grupo 1. Rhyucophilu fonticolu, Schizopelexfestivu y Tinodes waeneri; Grupo 2. Silonella aurutu, Calamoceras marsupus, Hydropsyche infernalis, Stenophilux crossotus, Sericostoma vittutum; Grupo 3. Polycentropus kingi, Rhyucophila mundu, Hydropsyche instubilis y Adicellu reducta; Grupo 4. Chimarru marginata, Hydropsyche cf. punicu y Setodes argentipunctellus; Grupo 5. Hydroptilu vectis y Hydropsyche lobatu y Grupo 6. Cheumutopsyche lepida, Hydropsyche exocellutu, Puduniella vandeli, Ecnomus deceptor, Ceruclea dissimilis y Cyrnus monserruti. A partir del análisis del biplot representado en la figura 5 pueden extraerse los condicionantes medioambientales que controlan la distribución de cada Asociación. La Asociacion I caracteriza los arroyos de cabecera con aguas siliceas; la Asociacion II es propia de los arroyos de cabecera, independiente el grado de mineralización del agua; la Asociación III esta relacionada, preferentemente, con el tramo de Benamahoma ( L4 ); la Asociación 


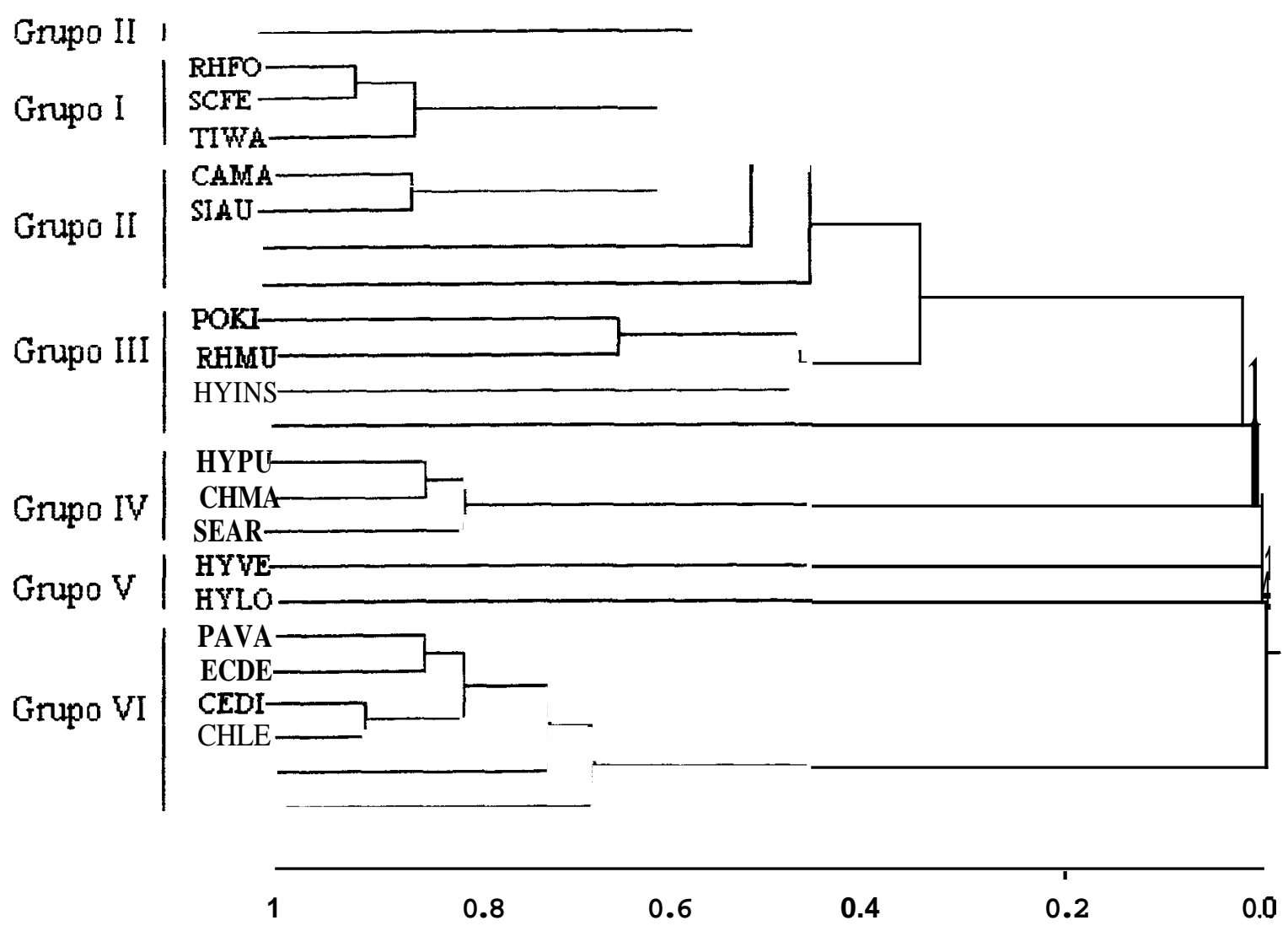

Figura 4. Agrupaciones obtenidds a partir de los inventarios de especies. Código asignado a cada especie. Clustering of species in lists. Code assigned to each species: RHFO Rhyacophila fonticola, PAVA Paduniella vandeli, RHMU Rhyacophila munda, TIWA Tinodes waeneri, HYVE Hydroptila vectis, ECDE Ecnomus deceptor, CHMA Chimurru marginata, STCR Stenophilax crossotus, CHLE Cheumatopsyche lepida, SIAU Silonella aurata, HYLO Hydropsyche lobata, CEDI Ceraclea dissimilis. HYEX Hydropsyche exocellata, SEAR Setodes argentipunctellus, HYIN Hydropsyche infernalis, ADRE Adicella reducta, HYINS Hydropsyche instabilis, SCFE Schizopelex festiva, HYPU Hydropsyche of punica, SEVI Sericostoma vittatum, POKI Polycentropus kingi, CAMA Calamoceras marsupus, CYMO Cyrnus monserrati.

IV se desarrolla en aquellos tramos altos, bien estructurados y donde se registran los mayores valores de diversidad; la Asociacion V está formada por aquellas especies generalistas sin preferencias ecologicas marcadas o ligadas a algún habitat en concreto; la Asociacion VI se localiza en el curso medio del Guadalete y en los tramos bajos algo degradados o con aguas salinas. Algún componente de este grupo (p. e. $H$. exocelleta) puede soportar condiciones de polucion orgánica (Verneaux \& Faessel, 1976; Gonzalez del Tánago \& García de Jalón, 1984).

\section{DISCUSIÓN}

Del análisis de las variables físico-químicas se desprende que la altitud, la permanencia del flujo (responsable de una mayor riqueza especifica) y la conductividad son los mejores parametros discriminantes de la distribución de las especies. Estas observaciones concuerdan con los resultados obtenidos previamente en un estudio del macrobentos de la parte alta de la cuenca del Guadalete donde los mejores parametros fueron la salinidad (medida como conductividad, cloruro 


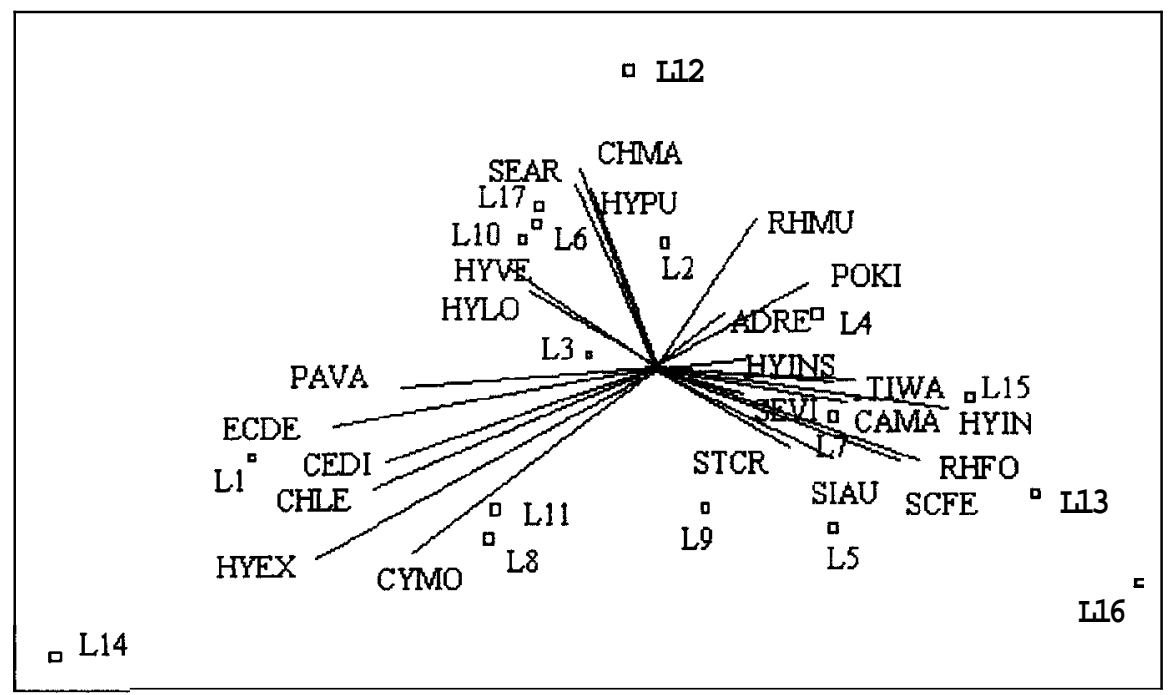

Figura 5. Situación de las especies y localidades en el espacio definido por los dos primeros ejes del PCA (biplot). Sampling sires und species location in the spacedefined by thefirst two axis of the Principal Component Analysis (PCA)biplot.

y sulfato), la velocidad de la corriente y la altitud (Gallardo-Mayenco, 1991).

La ordenación de las muestras nos permite establecer una clasificación de los cursos de agua estudiados (Fig. 3). En primer término se pueden diferenciar los tramos de cabecera de carácter permanente, con aguas frias ( temperatura inferior a 20 "C), alta diversidad y mineralización muy variada en función del sustrato geológico, de los cauces temporales caracterizados por comunidades pioneras de ciclo corto donde domina el tricóptero Stenophilux crossotus. Es de destacar la ausencia de Mesophilux aspersus, especie caracteristica de estos habitat (González del Tánago \& Garcia de Jalón, 1987), en el nacimiento del Guadalete, aunque han sido recogidos ejemplares en estado larvario en arroyos próximos de caracteristicas ecológicas semejantes. En la cabecera de los arroyos permanentes dominan las Asociaciones I y II, caracterizadas por las especies Rh.fonticola, Sc. festiva y $T$. waeneri, en el caso de arroyos siliceos; $S$. aurata, C. marsupus, $H$. infernalis en los arroyos tanto siliceos como calcareos y S. vittutum sólo en arroyos calcáreos. La Asociación III parece estar relacionada, principalmente, con la estación L4. El rio del Bosque constituye un ecosistema singular dentro del Area de estudio, representando el Rhythron según la terminologia de Illies \& Botosaneanu, 1963, con aguas frias (inferiores a 20 "C todo el año), velocidad de la corriente alta y caudales mucho más elevados que el resto de los arroyos de cabecera, que se mantienen durante todo el ciclo hidrológico. Sustenta una importante diversidad de tricópteros reófilos (3.82 bit/ind.), destacando $R h$ munda, $H$. instabilis, $A$. reducta y $P$. kingi. La distribución de esta ultima especie parece apoyar las observaciones de Gallardo-Mayenco, 1991, que la asocia a puntos en los que circula el agua todo el aiio y contradice los resultados de González del Tánago \& Garcia de Jalón, 1987 para ríos de Malaga, que la situan en arroyos temporales.

A menor altitud la pendiente se hace mas suave, los cauces y los caudales adquieren mayor entidad $\mathbf{y}$ se produce una diversificación del habitat que posibilita la proliferación de un mayor numero de especies. Esta imagen refleja donde las comunidades son más ricas; los lugares donde las condiciones para el establecimiento de 
una comunidad diversa son las mejores (Millet \& Prat, 1984 ). Estos tramos altos (Fig. 3 ) se caracterizan por la presencia de la Asociación IV, compuesta por Ch. marginata, S. argentipunctellus e H. cf. punica. No obstante, Gallardo-Mayenco et al., 1998 destacan el comportamiento oportunista de $H$. cf punica , que es capaz de colonizar hábitat temporales. En los ríos de Malaga Ch. marginata habita las cabeceras en altitudes medias y los tramos medios conviviendo siempre con $H$. exocellata (Garcia de Jalon \& Gonzalez del Tánago, 1986); en la cuenca del Sil ambas especies, junto con otros Hidropsiquidos, son caracteristicas de tramos bajos del rio y sus tributarios, afectados por polución orghnica, especialmente en verano ( De Soto et al., 1994), mientras que en este estudio estas dos especies pertenecen a dos asociaciones diferentes.

Las estaciones de Algodonales, Puerto Serrano y Junta de los Rios ilustran la situación del tramo medio y bajo del río Guadalete, mientras que el río del Alamo representa un curso de agua de carácter temporal y salino. Dichos tramos se corresponden con la Asociación VI, formada por las especies $C h$. lepida, H. exocellata, P. vandeli, E. deceptor, $C$. monserrati y $C$. dissimilis. Son cauces caracterizados por los elevados valores de temperatura y conductividad de sus aguas 10 que posibilita el desarrollo de comunidades termofilas y tolerantes a la salinidad, de media y baja diversidad.

Los Hidropsiquidos constituyen una fracción del macrobentos muy importante en el procesamiento y circulación de la materia y energia en los ambientes acuáticos (Wallace \& Merritt, 1980). Gallardo-Mayenco et al., 1998 han establecido dos agrupaciones para los Hydropsychidue de la cuenca del Guadalete: un grupo de tramos altos compuesto por $H$. infernalis, $H$. instabilis e $H$. punica; y un grupo de tramos bajos compuesto por $C h$. lepida e H. exocellata. Segun nuestros datos $H$. infernalis seria dominante en los tramos más altos, permanentes y de pequeño caudal; $H$. instabilis dominaría en tramos más caudalosos comportándose como una especie rhythrobionte (Verneaux \& Faessel, 1976); mientras que $H$. cf punica mostraría una mayor plasticidad ecológica al habitar en zonas con mayor fluctuación de caudales, incluso aguas temporales (Gallardo- Mayenco et al., 1998).

\section{AGRADECIMIENTOS}

Parte de la bibliografia consultada en la elaboración de este estudio nos ha sido proporcionada por la Dra. Josefina De Soto Cabo y el Dr. Alfonso Gallardo-Mayenco.

\section{BIBLIOGRAFÍA}

ALBA-TERCEDOR, J., GONZALEZ, G. \& PUIG, M. A., 1992. Present level of knowledge regarding fluvial macroinvertebrate communities in Spain. Limnetica, 8: 231 - 241.

BASAGUREN, A. \& ORIVE, E., 1991. Los insectos Tricopteros como indicadores de la calidad del agua de los nos de Bizkaia. Cuenca del Nervion. Kobie, 20: 39-50

BOURNAUD, M., KECK, G. \& RICHOUX, P., 1980. Les prelevements des macroinvertebrates benthiques en tant que revelateurs de la physionomie d'une riviere. Annls. Limnol., 16 (1): 55-75.

DE SOTO, J., FERNÁNDEZ-ALÁEZ, M., LUIS-CALABUIG, E. \& FERNANDEZ-ALAEZ, C., 1994.Spatial and temporal distribution of the caddisfly (Trichoptera) communities of the Sil basin (NW Spain). Verh. Internat. Verein. Limnnl., 25: 1716-1722.

GALLARDO-MAYENCO, A., 1991. Respuesta de los macroinvertebrados fluviales a la salinidad. Tésis de Doctorado. Universidad de Sevila. 125 pp.

GALLARDO-MAYENCO, A., 1993. Macroinvertebrate associations in two basins of SW Spain. Arch. Hydrobiol., 127 (4): 473-483.

GALLARDO-MAYENCO, A., 1994. Freshwater macroinvertebrate distribution in basins with different salinity gradients (Guadalete and Guadaira river basins, SW Spain). Int. J. Salt Lake Res., 3: 75-91.

GALLARDO-MAYENCO,A., PRENDA, J. \& TOJA, J., 1998. Spatio-temporal distribution and ecological preferences of coexisting Hydropsychid species (Trichoptera) in two mediteranean river basins (S. Spain). Internat. Rev. Hydrobiol., 83 (2): 123-134.

GARCÍA DE JALON, D. 1982.Contribución a la zoogeografia de los Tricópteros españoles. Shilap, Rev. Lepid. 10 (37, 38, 39): 75-78, 147-155, 237- 244. 
GARCÍA DE JALON, D. \& M. GONZALEZ DEL TANAGO, 1986. Ephemeroptera, Plecoptera y Trichoptera de los principales rios de Málaga. II Simp. del agua en Andalucia: 331-346.

GONZALEZ, M. A, D. GARCfA DE JALON \& L. TERRA, 1987. Faunistic studies on the Iberian Trichoptera: A historical survey and present state of knowledge. Proc. 5th. Int. Symp. Trichoptera, M. Bournaud \& H. Tachet (Eds.): 85-90.

GONZALEZ, M. A. \& MALICKY, H., 1988. Description de quatre nouvelles espèces de Trichoptkres de l'Espagne et du Maroc (Trichoptera). Mitt. Ent. Ges. Basel, 38 (2/3): 66-71. GONZALEZ, M. A. \& IGLESIAS, J. C., 1989. Une nouvelle espece de Trichoptkre du Sud de l'Espagne: Oecetis grazalemae (Trichoptera: Leptoceridae). Annls. Limnol., 25(1): 69-71.

GONZALEZ, M. A., COBO, F. \& IGLESIAS, J. C., 1990. Observaciones sobre los Tricópteros de la Peninsula Ibérica. IX. Provincias de Cadiz y Huelva. Bol. Asoc. Esp. Entom., 14: 207-214.

GONZALEZ DEL TANAGO, M. \& GARCIA DE JALON, D., 1984. Desarrollo de un índice biológico para estimar la calidad de las aguas de la cuenca del Duero. Limnetica, 1:263-272.

GONZALEZ DEL TANAGO, M. \& D. GARCfA DE JALÓN,1987. Clasificación de los rios de Malaga segun las comunidades del macrobentos. Actas IV Congreso español de Limnologia, Sevilla, España: 251-259.
ILLIES, J. \& BOTOSANEANU, L., 1963. Problemes et methodes de la classification et de la zonation écologique des eaux courantes, considerées surtout du point de vue faunistique. Mitt. int. Verein. theor. angew. Limnol., 12: 1-57.

MALICKY, H., 1979. Notes on some caddisflies (Trichoptera) from Europe and Iran. Aquatic Insect., 1 (1): 3-16.

MALICKY, H., 1982. Neur kocherfliegen (Trichoptera) aus Tunesien, Espanien und dem Iran. Ent. Zeit., 92 (15): 210-216.

MILLET, X. \& PRAT, N., 1984. Las comunidades de macroinvertebrados a 10 largo del río Llobregat. Limnetica, 1: 222-233.

PRENDA, J. \& GALLARDO-MAYENCO, A., 1999. Distributions patterns, species assemblages and habitat selection of the stoneflies (Plecoptera) from two mediterranean river basins in Southern Spain. Internat. Rev. Hydrobiol., 84 (6): 595-608.

RUIZ, A., 1998. Contribución a la biogeografia de los tricopteros (Insecta: Trichoptera) en Andalucia. Provincia de Cádiz. Rev. Soc. Gad. Hist. Nat., 1: 33-37.

VERNEAUX, J. \& FAESSEL, B., 1976. Larves du genre hydropsyche (Trichoptera: Hydropsychidae). Taxonomie, données biologiques et écologiques. Annls. Limnol., 12: 7-16.

WALLACE, J. B. \& MERRITT, R. W., 1980. Filterfeeding ecology of aquatic insects. Ann. Rev. Entomol., 25: 103-132. 